\title{
Characterization and co-expression analysis of WRKY orthologs involved in responses to multiple abiotic stresses in Pak-choi (Brassica campestris ssp. chinensis)
}

\author{
Jun Tang, Feng Wang, Zhen Wang, Zhinan Huang, Aisheng Xiong and Xilin Hou*
}

\begin{abstract}
Background: The WRKY transcription factor is an important member of the stress-related transcription factors, which mediate diverse abiotic stresses in many plants. However, up until now, the number of WRKY members, and the regulatory mechanisms involved in abiotic stress responses in Pak-choi (Brassica campestris ssp. chinensis), remained unknown.

Results: We isolated and identified 56 full-length WRKY cDNAs from a Pak-choi stress-induced cDNA library. The 56 putative BcWRKY proteins were divided into three groups based on structural and phylogenetic analyses. A subcellular localization prediction indicated that the putative BcWRKY proteins were enriched in the nuclear region. Experiments involving BCWRKY25 and BCWRKY40 confirmed the prediction. A total of 22 BCWRKYs were differentially expressed in response to at least one stress condition (abscisic acid, cold, salinity, heat, or osmosis) tested on Pak-choi leaves, and a co-expression analysis indicated stress-inducible BcWRKYs co-regulated multiple abiotic stresses. BCWRKY33, BCWRKY40, $B C W R K Y 53$, and BCWRKY70 acted as key regulators and played dominant roles within co-regulatory networks of stress-inducible BCWRKYs.

Conclusions: We first isolated and characterized the 56 stress-inducible WRKY transcription factor family members. A total of 22 stress-inducible BcWRKYs found in leaves can co-regulate multiple environmental stresses by integrating the potential mutual interactions of WRKYs in Pak-choi. This information will be valuable when exploring the molecular mechanisms of WRKYs in response to abiotic stresses in plants.
\end{abstract}

Keywords: WRKY transcription factor, Abiotic stress, Co-expression analysis, Subcellular localization, Pak-choi

\section{Background}

To overcome environmental stresses, plants have developed the ability to perceive and respond to these diverse external signals using specialized physiological and biochemical strategies [1,2]. Plant stress responses are generally controlled by a network of specialized genes that are intricately regulated by specific transcription factors (TFs) [3]. The WRKY TFs are important members of the stress-related TFs involved in regulating the plant's environmental stress responses [4-6]. The WRKY TF

\footnotetext{
* Correspondence: hxl@njau.edu.cn

State Key Laboratory of Crop Genetics and Germplasm Enhancement, College of Horticulture, Nanjing Agricultural University, Nanjing 210095, China
}

\section{Biomed Central}

(C) 2013 Tang et al.; licensee BioMed Central Ltd. This is an open access article distributed under the terms of the Creative Commons Attribution License (http://creativecommons.org/licenses/by/2.0), which permits unrestricted use, distribution, and reproduction in any medium, provided the original work is properly cited. family was named based on the presence of a 60 amino acid (aa) WRKY domain that is defined by a highly conserved WRKYGQK heptapeptide at the N-terminus and a zinc-finger-like motif at the C-terminus. WRKY proteins can be classified into three groups (I, II, and III) based on the number of WRKY domains and the pattern of the zinc-finger motif [5,7].

The WRKY TF family is a large conserved family of TFs that has been reported in many plants [8-15]. WRKY TFs have been found to be responsive to various abiotic stresses, including salinity, drought, cold, heat, and abscisic acid (ABA) signaling [16-20]. For instance, the $\mathrm{NaCl}$-inducible AtWRKY25 and AtWRKY33 mediate abiotic stresses [21]. AtWRKY63 is involved in plant 
responses to $\mathrm{ABA}$ and drought tolerance [22], and $A t$ WRKY34 mediates the cold sensitivity of mature pollen in Arabidopsis [23]. WRKY40, WRKY18, and WRKY60 interact with $\mathrm{ABAR}$ and negatively regulate $\mathrm{ABA}$ signaling [17]. Alleles of OsWRKY45-1 and OsWRKY45-2 play different roles in ABA signaling and salt stress tolerance in rice [24]. WRKY8 antagonistically interacts with VQ9 to modulate salinity stress tolerance [25]. Additionally, $B c$ WRKY46, a novel cold-inducible gene from Pak-choi (B. campestris ssp. chinensis, synonym of B. rapa ssp. chinensis) enhances the cold, salt and dehydration stress tolerance in transgenic tobacco [26].

Because of the functional complexity of the WRKY genes involved in environmental stresses, many approaches have been used to explore the unknown mechanisms of the stress response processes. Previous studies have demonstrated the power of co-expression analysis as a candidate discovery tool [27-29], which encouraged us to explore this approach for the identification of genes putatively involved in these interesting biological processes. Additionally, a co-expression analysis of OsWRKYS under biotic and abiotic stress conditions has been reported [30].

Pak-choi is an important Brassica crop with exceptional cold resistance [31], and its nearest genetic relative that has been sequenced is Chinese cabbage (B. rapa ssp. pekinensis) [32], which provides an effective model for Pak-choi research. Although WRKYs have been reported to mediate various stresses, the number of WRKY members in Pak-choi and their roles in response to abiotic stress tolerance were still unknown. Here, we cloned and identified 56 stress-inducible WRKY orthologs from Pak-choi, and we systematically investigated their organization, subcellular localization, and expression patterns under multiple abiotic stresses. In addition, we simultaneously measured co-expression of the stressinducible WRKY orthologs in Pak-choi and Arabidopsis thaliana. We subsequently established a co-regulatory network of stress-inducible $B c W R K Y$ s to multiple abiotic stresses, and indicated the possible interactions of stressinducible $B c W R K Y$ gene pairs.

\section{Results}

\section{Cloning stress-inducible BcWRKY genes from Pak-choi}

We isolated 56 BcWRKY genes from a multiple abiotic stress-treated Pak-choi cDNA library using a homology cloning method, which was based on sequence information from the $A$. thaliana WRKY gene family and the Chinese cabbage chiifu genome. We first designed degenerate and oligo (dT) primers to amplify the conserved regions of WRKY orthologs. Based on the PCR products' sequencing results, we designed gene-specific primers (Additional file 1: Table S1) and performed $5^{\prime}$-and $3^{\prime}$-RACE to amplify the full-length cDNA sequences of $B c W R K Y \mathrm{~s}$ from the stress-induced Pak-choi cDNA library. The stress-inducible
Pak-choi WRKY genes ranged from 531 to 3,195 base pairs (bp) and included 56 ORFs. These ORFs were confirmed by sequencing, and the sequences were submitted to GenBank (Table 1). The BcWRKY genes were named based on their similarity to the AtWRKY orthologs, and the molecular properties and sequence characteristics of the putative BcWRKY proteins were also analyzed. Among the $56 \mathrm{BcWRKY}$ proteins, the isoelectric point ranged from 4.69 to 10.45 , and the molecular weight ranged from 20.44 to $119.84 \mathrm{KDa}$ (Table 1).

\section{Phylogenetic analysis and identification of conserved motifs}

To investigate the phylogenetic relationship and structural features of the WRKY proteins in Pak-choi, an unrooted maximum likelihood (ML) phylogenetic tree and a linear distribution map of the conserved motifs in the putative BcWRKY proteins were produced (Figure 1). These were based on the multiple sequence alignment of the 56 putative BcWRKY proteins, ranging in size from 176 to 1,064 aa, using ClustalW in MEGA 5 software (using the ML method and a bootstrap value of 1000) and the MEME suite to detect conserved motifs in the BcWRKY protein sequences. The BcWRKYs were organized into three large clades, containing WRKY members that had the same or similar conserved motif distributions. The three clades were named I, II (IIa-e), and III, based on the number of WRKY domains and the type of zinc finger in the C-terminal WRKY domain. Group I consisted of 11 members containing two WRKY domains, while groups II and III contained 36 and nine members, respectively, and each member contained only one WRKY domain (Table 1). The distributions of different motifs formed groups, and the shared motifs appeared in all groups, such as motif 1 , motif 3 , and motif 5 (Figure 1 ). Of the eight motifs, motifs 1 and 3, which represented the distribution of C-or N-terminal WRKY domains, respectively, were both contained in the WRKY aa residues, and motif 5 contained nuclear localization signal (NLS) sequences, which could represent the NLS distribution of BcWRKY proteins (Table 2). Group I shared conserved motifs 1, 3, and 5, except for BcWRKY32, which lacked motif 3. Group II contained five subgroups (IIa-e) based on zinc finger types and consisted of 36 WRKY members, which contained several common and specific motifs, such as motifs 1,2 , and 4 that were shared by group II, and motifs 6 and 7 that were only found in group IIa and IIb (Figure 1).

\section{Alignment and comparison of WRKY domains}

To compare phylogenetic relationships among the WRKY domains, 67 WRKY domains that contained the highly conserved N-terminal WRKYGQK motif and C-terminal zinc finger were extracted and aligned from $56 \mathrm{BcWRKY}$ 
Table 1 Identification of stress-inducible WRKY genes in Pak-choi

\begin{tabular}{|c|c|c|c|c|c|c|c|c|c|c|}
\hline Gene name & Accession number & CDS (bp) & Size (aa) & Mass (KDa) & $\mathrm{pl}$ & Group & Atortholog & NLS locaton & NucPred score & $\begin{array}{c}\text { Nuclear } \\
\text { localization }\end{array}$ \\
\hline BCWRKY1 & KF430025 & 858 & 285 & 31.75 & 7.64 & \|la & AtWRKY18 & $95-98$ & 0.85 & Nucl: 13.0 \\
\hline BCWRKY2 & KF430026 & 2067 & 860 & 93.24 & 7.53 & I & AtWRKY2 & $152-170$ & 0.84 & Nucl: 14.0 \\
\hline BCWRKY3 & KF430027 & 1428 & 475 & 51.92 & 7.94 & 1 & AtWRKY3 & & 0.86 & Nucl: 13.0 \\
\hline BCWRKY4 & KF430028 & 1488 & 495 & 53.79 & 8.01 & 1 & AtWRKY4 & $339-351$ & 0.89 & Nucl: 14.0 \\
\hline BCWRKYG & KF430029 & 1662 & 550 & 60.34 & 5.94 & $11 \mathrm{~b}$ & AtWRKY6 & $267-268$ & 0.63 & Nucl: 13.0 \\
\hline BCWRKY7 & KF430030 & 1047 & 348 & 38.02 & 10.42 & Ild & AtWRKY7 & $253-273$ & 0.82 & Nucl: 12.0 \\
\hline BCWRKY8 & KF430031 & 966 & 379 & 42.58 & 7.14 & $\| c$ & AtWRKY8 & $189-226$ & 0.72 & Nucl: 13.0 \\
\hline BCWRKY9 & KF430032 & 987 & 328 & 37.38 & 5.55 & $11 \mathrm{~b}$ & AtWRKY9 & $113-125$ & 0.75 & Nucl: 12.5 \\
\hline BCWRKY11 & KF430033 & 999 & 332 & 36.37 & 10.18 & Ild & AtWRKY11 & $226-249$ & 0.92 & Nucl: 14.0 \\
\hline BCWRKY12 & KF430034 & 657 & 218 & 24.62 & 8.73 & IIC & AtWRKY12 & $112-133$ & 0.49 & Nucl: 4.0 \\
\hline BCWRKY13 & KF430035 & 882 & 293 & 32.97 & 9.36 & IIc & AtWRKY13 & $169-201$ & 0.74 & Nucl: 13.0 \\
\hline BCWRKY15 & KF430036 & 960 & 319 & 34.71 & 10.40 & IId & AtWRKY15 & $217-237$ & 0.86 & Nucl: 11.0 \\
\hline BCWRKY18 & KF430037 & 969 & 317 & 35.69 & 8.32 & Ila & AtWRKY18 & $25-32$ & 0.94 & Nucl: 13.0 \\
\hline BcWRKY20 & KF430038 & 1608 & 535 & 58.64 & 6.96 & 1 & AtWRKY20 & & 0.89 & Nucl: 14.0 \\
\hline BCWRKY21 & KF430039 & 1020 & 339 & 38.04 & 10.28 & Ild & AtWRKY21 & $247-267$ & 0.95 & Nucl: 14.0 \\
\hline BcWRKYZ22 & KF430040 & 897 & 298 & 32.34 & 7.16 & lle & AtWRKY22 & $101-121$ & 0.66 & Nucl: 13.0 \\
\hline BCWRKYZ23 & KF430041 & 972 & 323 & 36.10 & 6.91 & IIC & AtWRKY23 & 133-152 & 0.74 & Nucl: 13.0 \\
\hline BCWRKY24 & KF430042 & 531 & 176 & 20.44 & 8.62 & Ilc & AtWRKY24 & $67-87$ & 0.5 & Nucl: 6.0 \\
\hline BcWRKY25 & KF430043 & 1122 & 373 & 42.06 & 6.68 & I & AtWRKY25 & $206-223$ & 0.58 & Nucl: 13.0 \\
\hline BCWRKY26 & KF430044 & 894 & 319 & 35.94 & 9.62 & I & AtWRKY26 & $217-230$ & 0.64 & Nucl: 12.5 \\
\hline BCWRKY28 & KF430045 & 939 & 312 & 34.97 & 6.72 & IIC & AtWRKY28 & 116-156 & 0.69 & Nucl: 13.0 \\
\hline BCWRKY29 & KF430046 & 927 & 308 & 34.17 & 8.43 & lle & AtWRKY29 & 108-131 & 0.55 & Nucl: 13.0 \\
\hline BcWRKY 30 & KF430047 & 942 & 313 & 35.57 & 6.80 & III & AtWRKY30 & $97-104$ & 0.52 & Nucl: 11.5 \\
\hline BCWRKY31 & KF430048 & 1560 & 519 & 57.15 & 6.95 & $\| \mathrm{b}$ & AtWRKY42 & $212-217$ & 0.69 & Nucl: 14.0 \\
\hline BcWRKY32 & KF430049 & 1899 & 459 & 49.58 & 6.97 & 1 & AtWRKY26 & $300-311$ & 0.61 & Nucl: 11.0 \\
\hline BCWRKY33 & KF430050 & 1557 & 518 & 56.63 & 7.92 & 1 & AtWRKY33 & & 0.77 & Nucl: 13.0 \\
\hline BCWRKY34 & KF430051 & 1650 & 549 & 60.18 & 6.47 & 1 & AtWRKY34 & $19-28$ & 0.78 & Nucl: 14.0 \\
\hline BCWRKY36 & KF430052 & 1152 & 383 & 42.99 & 7.69 & $11 \mathrm{~b}$ & AtWRKY36 & $96-129$ & 0.74 & Nucl: 12.0 \\
\hline BCWRKY39 & KF430053 & 1029 & 342 & 38.00 & 10.02 & Ild & AtWRKY74 & $247-271$ & 0.9 & Nucl: 11.0, \\
\hline BCWRKY4O & KF430054 & 873 & 290 & 32.43 & 7.82 & Ila & AtWRKY40 & $75-89$ & 0.72 & Nucl: 10.0 \\
\hline BCWRKY42 & KF430055 & 1551 & 519 & 57.15 & 6.95 & $11 \mathrm{~b}$ & AtWRKY42 & $212-217$ & 0.69 & Nucl: 14.0 \\
\hline BCWRKY44 & KF430056 & 1230 & 409 & 44.89 & 9.19 & I & AtWRKY44 & $360-364$ & 0.67 & Nucl: 8.0 \\
\hline BcWRKY46 & KF430057 & 858 & 283 & 32.20 & 5.82 & III & AtWRKY46 & $60-90$ & 0.73 & Nucl: 10.0 \\
\hline BCWRKY47 & KF430058 & 1512 & 490 & 53.94 & 6.79 & $11 \mathrm{~b}$ & AtWRKY47 & & 0.77 & Nucl: 13.0 \\
\hline BCWRKY48 & KF430059 & 1200 & 399 & 44.46 & 6.77 & IIC & AtWRKY48 & $185-212$ & 0.69 & Nucl: 13.0 \\
\hline BCWRKY51 & KF430060 & 597 & 198 & 22.29 & 7.54 & IIC & AtWRKY51 & $112-129$ & 0.49 & Nucl: 12.0 \\
\hline BCWRKY53 & KF430061 & 972 & 323 & 36.19 & 6.38 & III & AtWRKY53 & 127-149 & 0.81 & Nucl: 14.0 \\
\hline BCWRKY54 & KF430062 & 1020 & 297 & 33.11 & 4.76 & III & AtWRKY54 & 107-131 & 0.8 & Nucl: 12.0 \\
\hline BCWRKY56 & KF430063 & 564 & 186 & 21.03 & 7.23 & Ilc & AtWRKY56 & $79-89$ & 0.37 & Nucl: 9.0 \\
\hline BCWRKY57 & KF430064 & 885 & 294 & 32.56 & 6.89 & Ilc & AtWRKY57 & $116-141$ & 0.67 & Nucl: 13.0 \\
\hline BcWRKY58 & KF430065 & 3195 & 1082 & 119.84 & 9.63 & 1 & AtWRKY58 & 865-913 & 0.96 & Nucl: 14.0 \\
\hline BCWRKY59 & KF430066 & 546 & 196 & 22.64 & 6.95 & IIc & AtWRKY59 & $75-97$ & 0.84 & Nucl: 5.0 \\
\hline BCWRKY 60 & KF430067 & 927 & 308 & 34.71 & 8.22 & Ila & AtWRKY18 & 103-109 & 0.91 & Nucl: 13.0 \\
\hline
\end{tabular}


Table 1 Identification of stress-inducible WRKY genes in Pak-choi (Continued)

\begin{tabular}{|c|c|c|c|c|c|c|c|c|c|c|}
\hline BCWRKYG1 & KF430068 & 1662 & 398 & 44.24 & 6.44 & $11 \mathrm{~b}$ & AtWRKY61 & & 0.66 & Nucl: 12.0 \\
\hline BcWRKY 62 & KF430069 & 837 & 233 & 26.92 & 6.27 & III & AtWRKY62 & $57-73$ & 0.61 & Nucl: 13.0 \\
\hline BcWRKY 64 & KF430070 & 777 & 258 & 29.36 & 4.82 & III & AtWRKY67 & $108-117$ & 0.67 & Nucl: 11.0 \\
\hline BCWRKY 65 & KF430071 & 786 & 261 & 29.25 & 5.27 & lle & AtWRKY65 & 133-150 & 0.55 & Nucl: 13.0 \\
\hline BCWRKY 66 & KF430072 & 738 & 452 & 52.12 & 6.96 & III & AtWRKY64 & $121-138$ & 0.56 & Nucl: 8.0 \\
\hline BCWRKY 67 & KF430073 & 831 & 276 & 31.06 & 6.79 & III & AtWRKY70 & $92-118$ & 0.81 & Nucl: 11.0 \\
\hline BCWRKY 68 & KF430074 & 960 & 319 & 36.04 & 6.61 & IIC & AtWRKY23 & $116-145$ & 0.82 & Nucl: 13.0 \\
\hline BCWRKY 69 & KF430075 & 849 & 282 & 31.27 & 4.69 & lle & AtWRKY69 & $32-41$ & 0.46 & Nucl: 13.0 \\
\hline BCWRKY7O & KF430076 & 852 & 283 & 32.01 & 6.23 & III & AtWRKY70 & $92-117$ & 0.77 & Nucl: 12.0 \\
\hline BCWRKY71 & KF430077 & 1047 & 277 & 31.53 & 8.45 & Ilc & AtWRKY71 & $99-125$ & 0.46 & Nucl: 11.0 \\
\hline BCWRKY72 & KF430078 & 2529 & 551 & 59.68 & 6.36 & $\mathrm{llb}$ & AtWRKY72 & & 0.58 & Nucl: 14.0 \\
\hline BCWRKYY4 & KF430079 & 969 & 322 & 35.29 & 10.45 & Ild & AtWRKY11 & $211-244$ & 0.96 & Nucl: 13.0 \\
\hline BCWRKY75 & KF430080 & 1062 & 353 & 39.33 & 6.85 & $\| c$ & AtWRKY75 & $143-167$ & 0.73 & Nucl: 13.0 \\
\hline
\end{tabular}

Abbreviations: bp, base pair; aa, amino acids; CDS, coding sequence; pl, Isoelectric point; WD, WRKY domain; NLS, Nuclear location signal. NLS location indicated the distribution of the NLSs on the BcWRKY proteins.

proteins. The WRKY domains were classified into the eight subgroups and named ICT, INT, IIa-e, and III (Figure 2 and Additional file 2: Figure S1). Eleven members of group I, which contained two WRKY domains, including a C-or N-terminal WRKY domain, were separately divided into groups INT and ICT. The 36 WRKY domains of group II each contained one WRKYGQK motif and a $\mathrm{C}_{2} \mathrm{C}_{2}$-type zinc finger motif $\left(\mathrm{C}-\mathrm{X}_{5}-\mathrm{C}-\mathrm{X}_{23}-\mathrm{H}-\mathrm{X}_{1}-\mathrm{H}\right)$, and could be classified into five distinct subgroups (IIa-e) based on different conserved motifs contained in the WRKY domain. Group IIa was comprised of $B c W R K Y 1$, BcWRKY18, BcWRKY40, and BcWRKY60, which each contained motifs 1,2 , and 4 in the WRKY domain and motifs 6 and 7 outside of the WRKY domain. Group IIb had nine members that each contained motifs 1,2 , 4, and 5 in the WRKY domain and motifs 6 and 8 outside of the WRKY domain. Group IIc and IId had 14 and eight members, respectively, and contained motifs $1,2,4$, and 5 in the WRKY domains, but the motif positions were different. The conserved motifs of group IId only occurred at the C-terminus of the WRKY proteins. The group IIe WRKY domains only contained motifs 1, 2, and 4 (Figure 1). Group III had nine WRKY members that only shared motifs 1 and 2, and whose WRKY domains contained the C2HC-type (C-X7-C-X23-H-X1-C) of zinc finger motif (Additional file 2: Figure S1). The patterns of WRKY domains and zinc finger motifs in the BcWRKY proteins were similar to the patterns of Arabidopsis WRKY domains (Additional file 2: Figure S1) and were consistent with a previous study on groups II $\left(\mathrm{C}^{-\mathrm{X}_{4-5^{-}}}\right.$ $\left.\mathrm{C}-\mathrm{X}_{22-23}-\mathrm{H}-\mathrm{X}_{1}-\mathrm{H}\right)$ and III $\left(\mathrm{C}-\mathrm{X}_{7}-\mathrm{C}-\mathrm{X}_{23}-\mathrm{H}-\mathrm{X}_{1}-\mathrm{C}\right)[4]$.

By comparing the 67 WRKY domains, a phylogenetic tree with five clades was constructed (Figure 2). In terms of the eight WRKY domain patterns, the ICT group was placed in clade I, and the next branch, clade II, consisted of group IIc. This was considered as an intermediate between clades I and III, which involved groups ICT and INT. While clade III, including the INT group, was placed as an original node, the remaining 31 domains were clustered into clade IV (groups IIa and IIb) and clade V (IId, IIe, and III). These results illustrated the evolution of Pak-choi WRKY domains from group I to group II or III (Figure 2). For example, BcWRKY51 and BcWRKY59, belonged to group IIc, but they were clustered into the INT group in the phylogenetic tree (Figure 2), and BcWRKY32 protein belonged to group I, containing two WRKY domains, but clustered into the IIc group in the phylogenetic tree (Figure 1). A similar evolutionary pattern for WRKY domains has been reported in Arabidopsis and rice [8].

\section{Subcellular localization analysis of BcWRKYs}

To investigate the subcellular localization of putative BcWRKY proteins, we used NLStradamus with the default settings. We found $50 \mathrm{BcWRKY}$ proteins contained NLSs. Additionally, we used NucPred and WOLF PSORT to predict the nuclear localization scores of the BcWRKY proteins. Fifty BcWRKY proteins had a NucPres-score of $\geq 0.5$ and 53 had nuclear localization scores of $\geq 7 \quad(\mathrm{KNN}=14)$ using WOLF PSORT (Table 1). A consensus of the results generated predicted that most BcWRKYs $(47 / 56)$ localized at the nucleus (Figure 3A). Additionally, we used a transient expression system in onion epidermal cells to test the subcellular localization of BcWRKY proteins. The yellow fluorescent marker protein (YFP) was fused to BcWRKY25 and BcWRKY40 and the expression of the fusion genes was tracked by the marker's signal (Figure 3B). When YFP alone was expressed the fluorescence was observed in the cytosol and nucleus (Figure 3C, upper panel), while the yellow fluorescence 


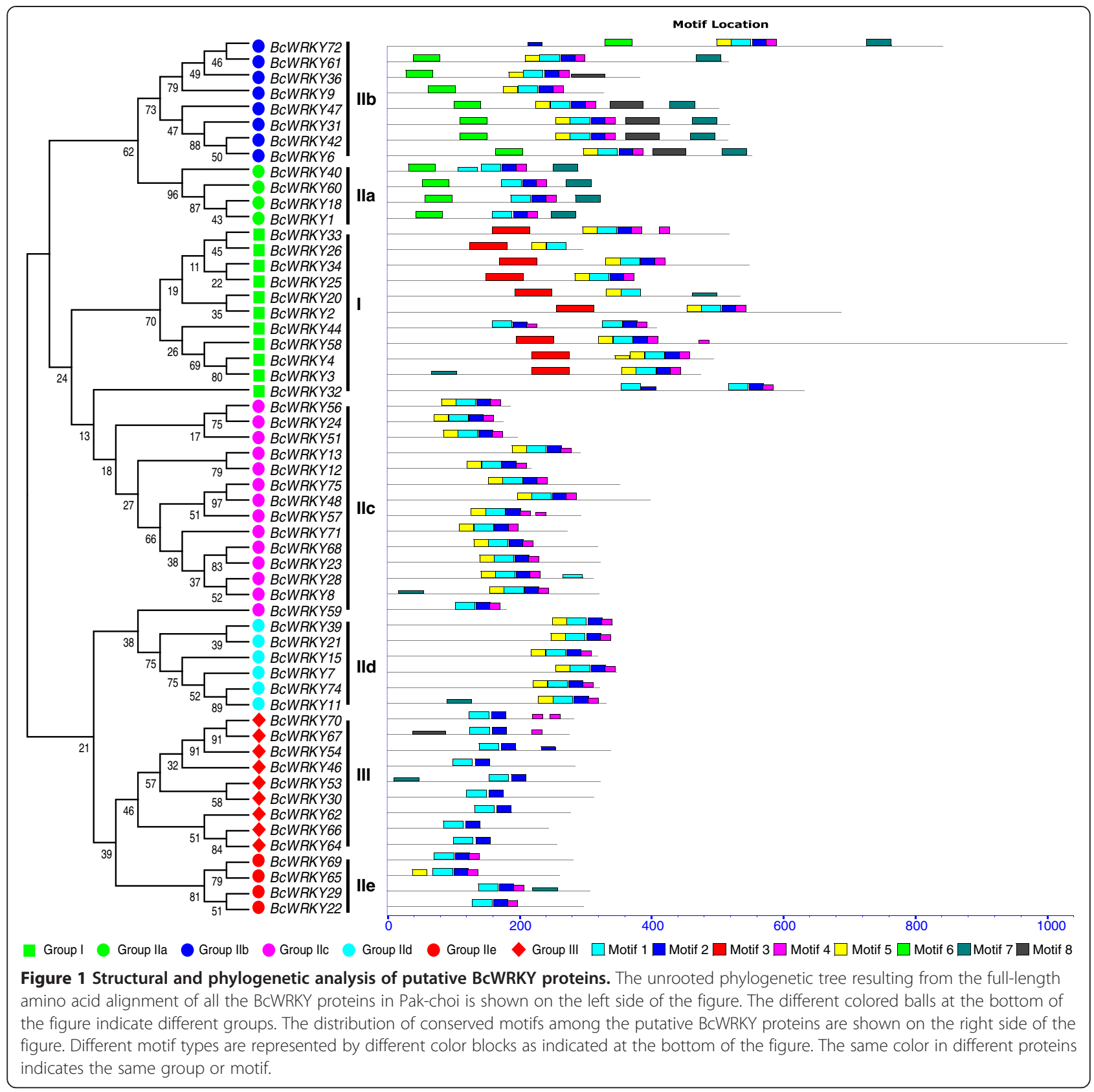

Table 2 Motif sequences of 56 BcWRKY proteins identified by MEME tools

\begin{tabular}{lll}
\hline Motif & Consensus sequence & Known motif description \\
\hline Motif1 & LDDGYRWRKYGQKPVKGSPYPRSYYRCTT & WRKY \\
Motif2 & CPVRKQVERSAEDPSIVITTY & WRKY \\
Motif3 & NDGYQWRKYGQKVAKGNPCPRAYYRCTMA & Nuclear location signal \\
Motif4 & EGKHNHPLPXARXSXASSTSA & \\
Motif5 & KKSEKKVREPRVAVQTRSDVD & \\
Motif6 & LREELNRVNEENKKLKEMLSQVXENYNSLQMHLEKLMRQQ & \\
Motif7 & MQEVLVEQMASALTADPNFTAALAAAISSIIGGQNNT & \\
Motif8 & KFTQGCKATKQVQKIENDPSLFRITYIGKHTCNV & \\
\hline
\end{tabular}

Significant motifs (e-value $<1$ e-100) of more than 10 amino acid length were predicted by MEME search. 


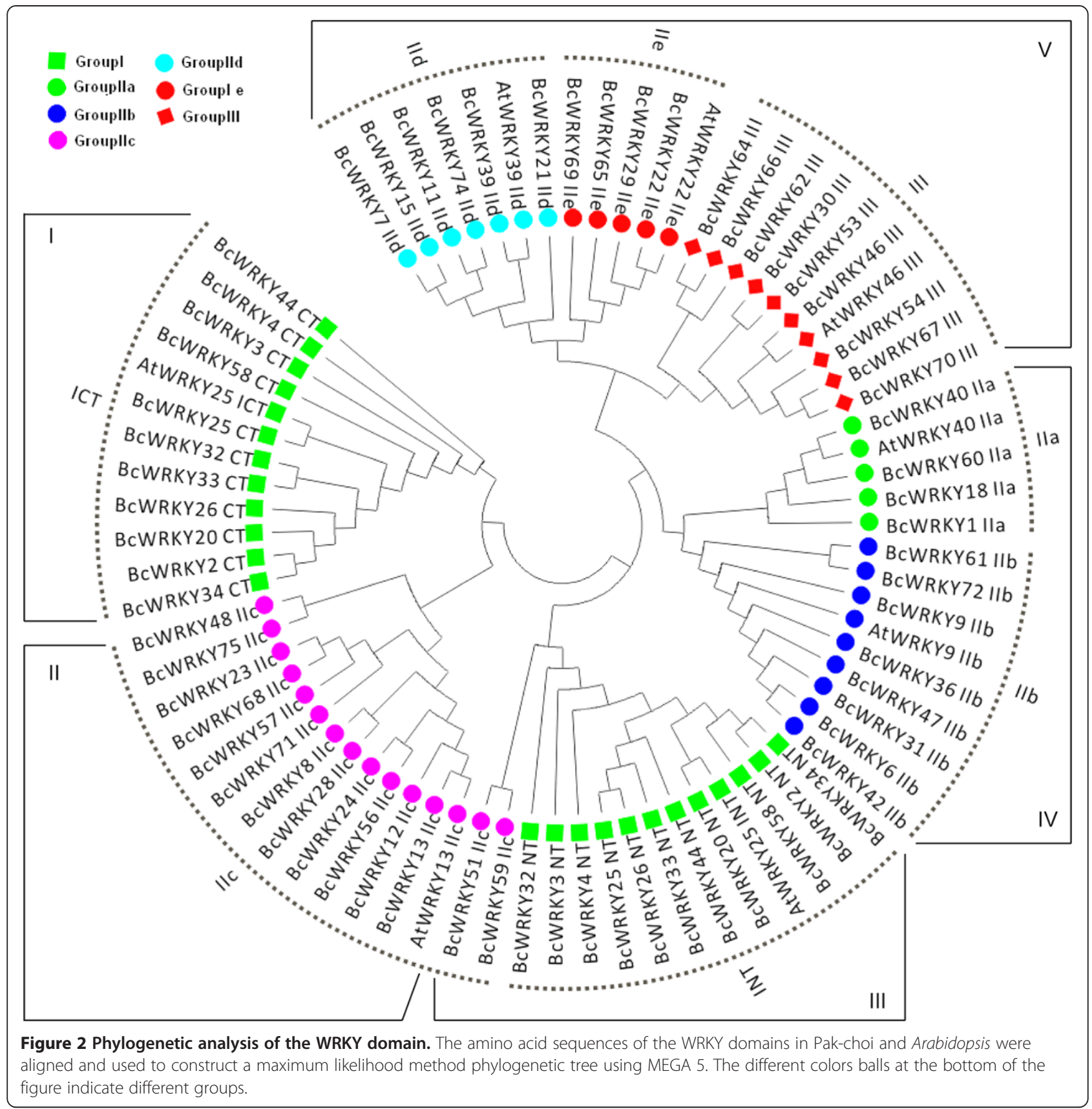

of the BcWRKY25-YFP and BcWRKY40-YFP fusions were observed in the nuclear region (Figure $3 \mathrm{C}$, middle and lower panel, respectively). Thus, BcWRKY25 and 40 were localized to the nucleus, which agreed with the protein subcellular localization prediction. These results indicate that the properties of the BcWRKY proteins define them as transcription factors.

\section{Expression patterns of BcWRKYs under multiple} abiotic stresses

Among the 56 isolated WRKY genes, 22 genes were detected and found to be significantly induced in response to ABA and abiotic stresses in Pak-choi leaves. Of the 22 expressed stress-inducible BcWRKYs, 19 were up-regulated in at least one of the five treatments (ABA, salinity, cold, heat, and osmosis) and six genes were down-regulated under heat treatment (Figure 4). During multiple abiotic stress treatments, $B c W R K Y 25,40,60$, and 75 were all highly expressed during an $\mathrm{ABA}$ treatment time course. Similarly, BcWRKY25, 26, 34, 39, and 60 under cold stress treatments, had significantly upgraded expression levels. A heat treatment also strongly induced high expression levels in some genes, such as $B c W R K Y 25$, 26, 34, 39, and 60 (Figure 4). Additionally, BcWRKY2, 6, 


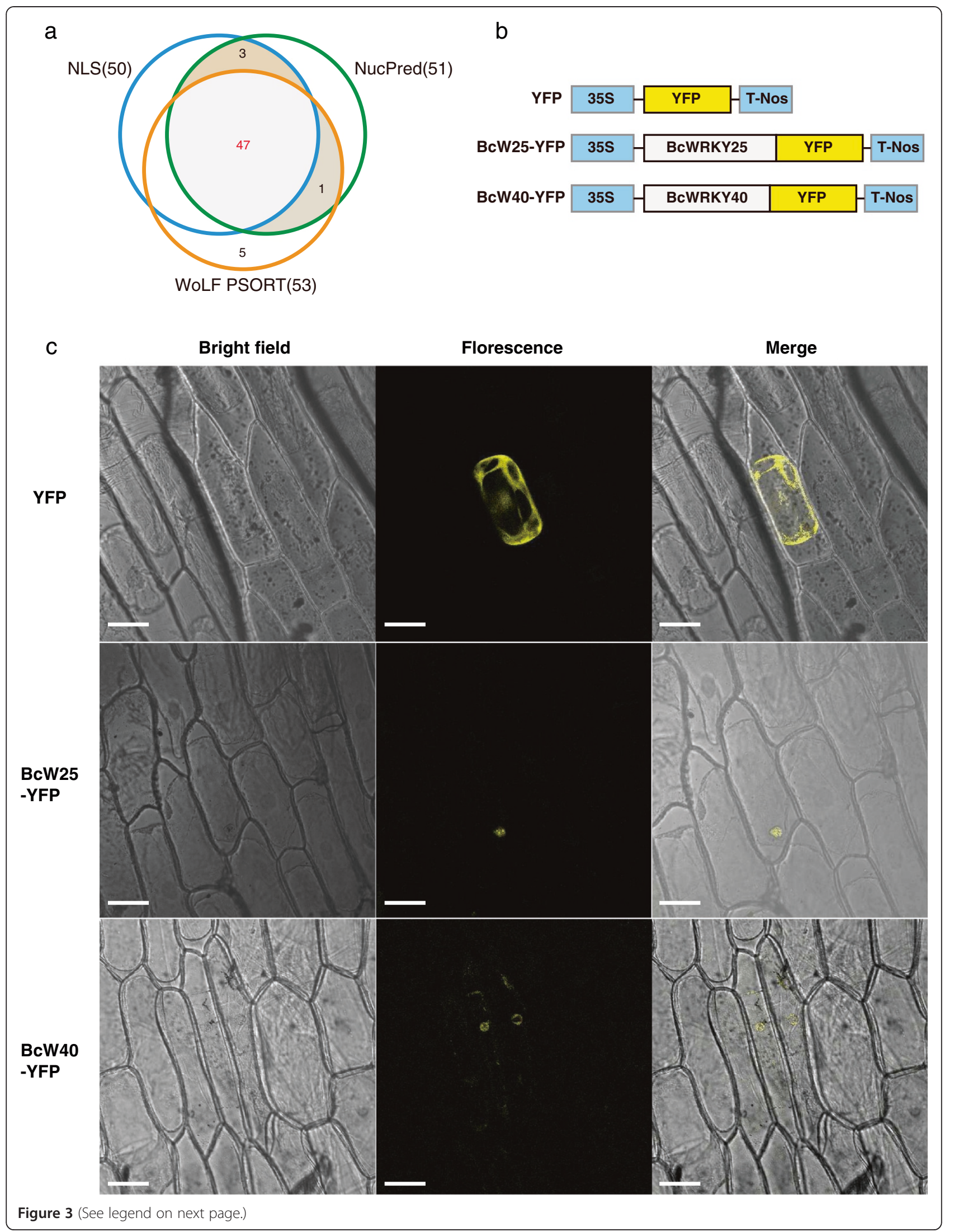


(See figure on previous page.)

Figure 3 Subcellular localization analysis of BcWRKYs. The subcellular localization analysis of the putative BcWRKY proteins used NLStradamus, NucPred, and WOLF PSORT with the default settings to detect nuclear localization scores. (A) two yellow fluorescent protein (YFP) marker expressing BCWRKY fusion genes, BcWRKY25-YFP and BcWRKY40-YFP, were constructed; (B) BcWRKY25-YFP and BcWRKY40-YFP were introduced into onion epidermal cells by particle bombardment with the YFP signal as an indicating marker to test the subcellular localization of BCWRKY proteins; (C) the upper panel, the corresponding bright field, fluorescence, merged fluorescence image of YFP control; the middle panel, the corresponding bright field, fluorescence, merged fluorescence image of BcW25-YFP; the lower panel, the corresponding bright field, fluorescence, merged fluorescence image of BcW40-YFP. Scale bars: $20 \mu \mathrm{m}$.

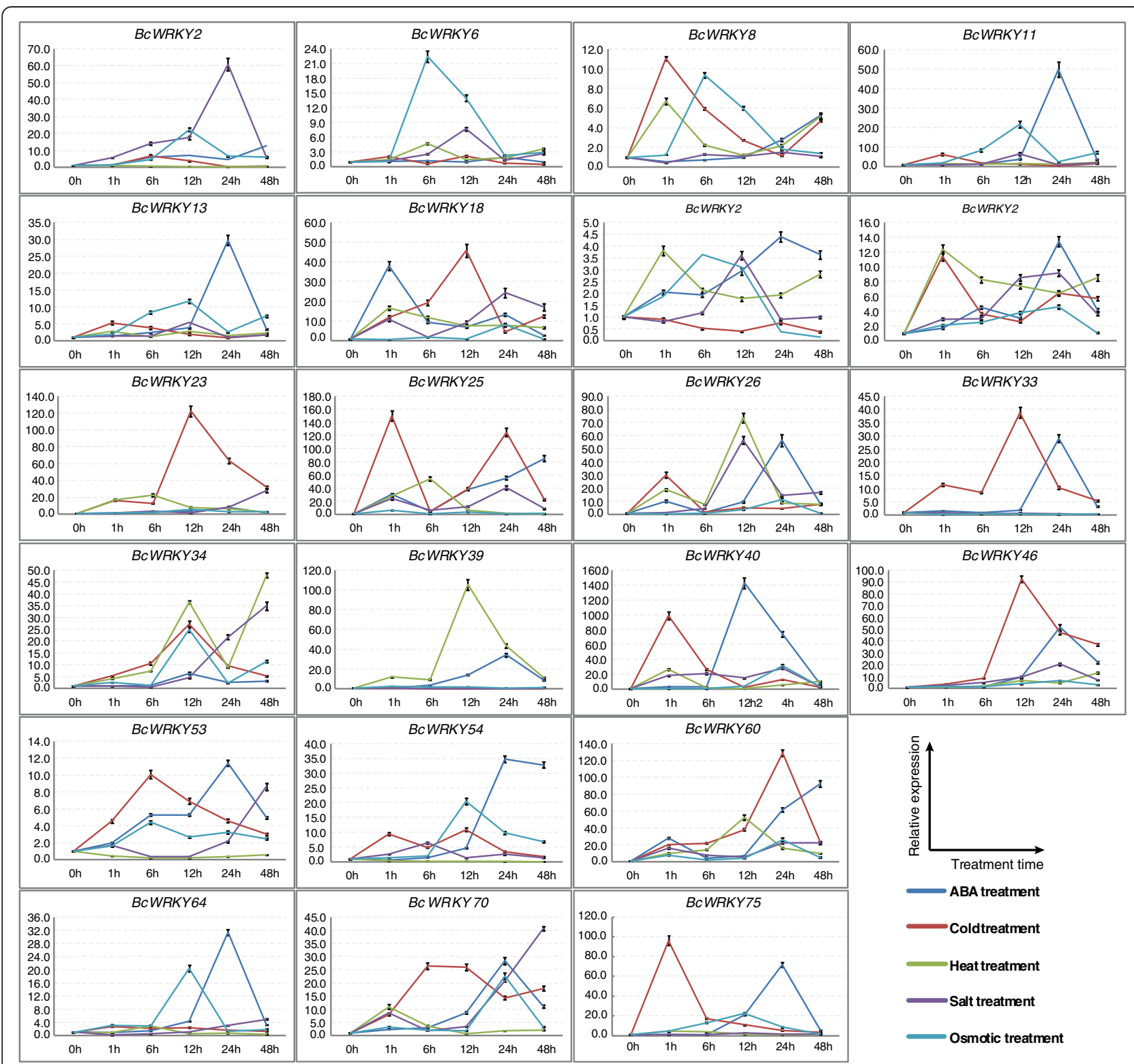

Figure 4 Expression patterns of the 22 BcWRKYs under abiotic stresses. Pak-choi plants were subjected to ABA, cold, heat, high salinity, and osmotic treatments, respectively. The 22 stress-inducible $B C W R K Y$ s were significantly expressed in response to multiple abiotic stresses in the leaves and their transcript levels were quantified against $B C G A P D H$ transcript levels using $2^{-\Delta \Delta C T}, \Delta \Delta C T=\Delta C T$ (treated sample) $-\Delta C T$ (untreated sample), $\Delta C T=\mathrm{CT}_{\text {target }}-\mathrm{CT}_{\text {BCGAPDH }}$. 
23, 26, 34, 40, 60 and 70 had high expression levels under salt treatment and osmotic treatment. Meanwhile, most $B c W R K Y \mathrm{~s}^{\prime}$ expression levels peaked at the 12-h or 24-h time-points. However, the expression peaks for $B c W R K Y 22,25,26$, and 40 occurred at the 1-h time-point and BcWRKY53 and BcWRKY70 peaked at the 6-h time-point under cold treatment (Figure 4). Interestingly, BcWRKY18, 25, 40, 60, 70 were all predominantly expressed in response to multiple stress treatments, and the simultaneous expression of the $B c W R K Y_{\mathrm{s}}$ was detected. These expression processes exhibited some low to high or high to low curve changes over the $48 \mathrm{~h}$ time course, showing that the inducible responses of $B c W R K Y \mathrm{~s}$ to multiple abiotic stresses is a dynamic process. The results indicated that stress-inducible $B c W R K Y \mathrm{~s}$ were strongly induced and coordinately mediated in response to multiple abiotic stresses in Pak-choi leaves.

\section{Co-regulatory networks of BcWRKYs in response to multiple abiotic stresses}

Co-regulatory networks were established based on the Pearson correlation coefficient of stress-inducible BcWRKY gene pairs using $\log _{2}$ transformed qPCR data (Figure 5). All Pearson correlations that were significant at the 0.05 significance level ( $\mathrm{p}$-value) were collected and visualized by Cytoscape 2.8 to construct stress co-regulatory networks of BcWRKYs. There were 22 nodes representing 22 stress-inducible $B c W R K Y_{\mathrm{S}}$ separately connected by 56 edges, which represented the Pearson correlation coefficients of the co-regulatory gene pairs (Figure 5). In the co-regulatory networks, most co-regulatory gene pairs (51/56) appeared to have positive significant correlations, except for four $B c W R K Y$ gene pairs, $B c W R K Y 2-B c W R K Y 39$, BcWRKY6-BcWRKY70, BcWRKY6-BcWRKY25, and BcWR KY6-BcWRKY33, which had negative correlations (0.05). In addition, $B c W R K Y 6-B c W R K Y 40$ had a large negative correlation at the 0.001 significance level. Among 51 positively correlated gene pairs, 27 and 29 gene pairs were significant at the 0.05 and 0.001 levels, respectively (Figure 5). Among the 22 mutually linked nodes, BrWTKY33, 40, 54, and 70 had more edges, with 10, 8, 7, and 10, respectively (Figure 5). These results suggested that they represented central nodes in the co-regulatory networks of BcWRKYS in response to multiple abiotic stresses. All data used to calculate the correlations are shown in Additional file 3: Table S3.

To validate the co-regulatory relationships of the $B c W R K Y$ genes, we constructed an evident interaction co-regulatory network of WRKY orthologs through the STRING 9.1 database based on the stress-inducible BcWRKY orthologs in Arabidopsis (Additional file 4: Figure S2). Among the 11 co-regulatory WRKY members, each of them existed in at least two mutual relationships with others in the given datasets (Databases, Text-mining, Homology, Experiments, Neighborhood, Co-occurrence, and Co-expression). Those were further verified, and they supported the co-regulatory networks of stress-inducible $B c W R K Y$ s in which WRKY33, 40, 53 and 70 represented central nodes.

\section{Discussion}

This study reported the isolation and identification of 56 stress-inducible $B c W R K Y$ genes (Table 1) using a homologous gene cloning method from a stress-induced Pak-choi cDNA library. We also systematically surveyed the structure, phylogeny, and conserved motifs of the BcWRKYs and measured the putative protein localization. In addition, a co-expression analysis of BcWRKYS was performed to explore the co-regulatory information of WRKY TFs. Based on a sequence alignment of the BcWRKY domains (Additional file 2: Figure S1), we clearly found phenomena similar to those previously reported [5]. This indicated that possessing different numbers of WRKY domains and zinc finger motifs, and structural variations of the WRKY domain, may produce some novel WRKY members (Additional file 2: Figure S1). For example, the BcWRKY32 protein contains two of the same type of WRKY domains, belonging to Group I; however, in a phylogenetic analysis it can be clustered together with Group IIc members, while BcWRKY59 with WRKY domain features of Group IIc was clustered into a clade with Group IId members because of the absence of some motifs (Figure 1). These results also support the belief that group I WRKYs may represent the ancestral form of the WRKY family [33]. Additionally, the conservation of WRKY domains implies a conserved function for WRKY TFs [34]. For example, the subcellular localization of BcWRKY25 and BcWRKY40 proteins was in the nucleus, which was appropriate for TFs, thus supporting their classification as functional TFs [35].

Although the mechanisms of the WRKYs' responses to multiple abiotic stresses needs further investigation, the co-expression analysis, which has the ability to measure large numbers of gene expressions, provides a powerful tool for identifying groups of genes and discovering novel regulators involved in the signal transport of plant stress responses. Previous studies have performed co-expression analyses $[29,30,36]$, and found many unknown relationships and novel genes encoding proteins involved in similar expression patterns under different conditions. The coexpression analysis of Arabidopsis and Pak-choi WRKY orthologs (Figure 5 and Additional file 4: Figure S2) indicated co-regulatory relationships and key regulators of 22 stress-inducible $B c W R K Y \mathrm{~s}$ in response to multiple abiotic stresses, and it further indicated the important roles of the WRKY TF mutual interactions to mediate 


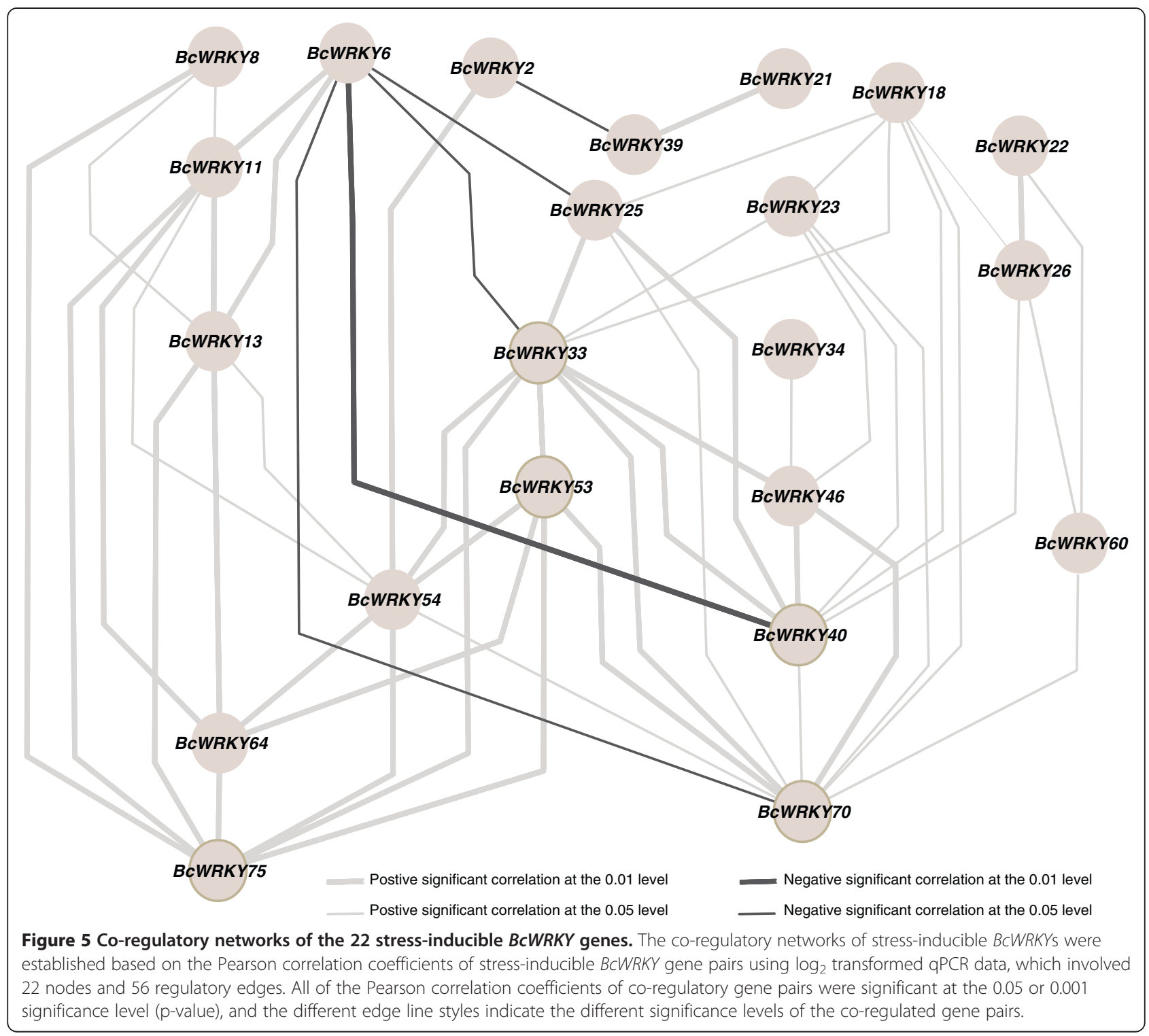

complex biological processes [37]. These findings will help in identifying and understanding more interactive relationships among the WRKYs and will elucidate more co-regulatory relationships for WRKYs under multiple abiotic stresses.

\section{Conclusions}

This study isolated and characterized 56 Pak-choi stressinducible WRKY genes, indicated that $22 B c W R K Y$ genes co-regulated multiple abiotic stress responses in Pak-choi leaves, and established a co-regulatory network of stressinducible $B c W R K Y$ s. The co-regulatory network showed that WRKY33, 40, 53 and 70 were central regulators and had potentially interactive relationships. This study also revealed a method of measuring the common and distinct functions of stress-inducible $B c W R K Y$ s among multiple abiotic stress responses, which may aid in exploring the molecular mechanisms of WRKYs in response to abiotic stresses in plants.

\section{Methods}

Plant materials, growth conditions and stress treatments Pak-choi (B. campestris ssp. chinensis cv. suzhonqing) was used for all experiments. Seedlings were soaked in distilled water for $0.5 \mathrm{~h}$, and then germinated in plastic Petri dishes containing filter paper saturated with distilled water in darkness at $22^{\circ} \mathrm{C}$ for 2 days. Seedlings were then transferred to $4 \mathrm{~L}$ hydroponic containers containing continuously aerated 1/2 Murashige and Skoog (MS) liquid solution ( $\mathrm{pH} 5.8$, without agar and sugar). The 1/2 MS liquid solution was changed once every 3 days. Three- 
week-old seedlings were transferred to new $1 / 2$ MS liquid solution ( $\mathrm{pH} 5.8$, without agar and sugar) for multiple stress treatments under a continuous time course $(0,1,6$, 12 , 24, and $48 \mathrm{~h}$ ). For ABA, salt and osmotic treatments, seedlings were exposed to $1 / 2$ MS solution ( $\mathrm{pH} 5.8$ ) containing $100 \mu \mathrm{M} \mathrm{ABA}, 200 \mathrm{mM} \mathrm{NaCl}$ and $15 \%$ (w/v) polyethylene glycol (PEG), respectively. For cold and heat treatments, seedlings were exposed to the 4 and $38^{\circ} \mathrm{C}$ conditions in $1 / 2 \mathrm{MS}$ solution $(\mathrm{pH} 5.8$ ), respectively. All seedlings were placed under the same growth conditions, except for the different treatment factors, and exposed to $1 / 2 \mathrm{MS}$ solution at $22^{\circ} \mathrm{C}$ as controls. The seedlings were harvested under a continuous time courses $(0,1,6,12,24$, and $48 \mathrm{~h})$ in three biological replicates for RNA preparation.

\section{Cloning and identification of the BcWRKY members in Pak-choi}

Total RNA was extracted from Pak-choi roots, stems, and leaves under multiple abiotic stress conditions using the RNAeasy mini kit (Tiangen, Beijing, China). A mixture of total RNA $(1 \mu \mathrm{g})$ was used for first-strand cDNA synthesis using a superscript II kit (Takara, Dalian, China) following the manufacturer's instructions to construct a stress-induced Pak-choi cDNA library. To clone BcWRKY genes, we first designed degenerate primers $\left(5^{\prime}-\mathrm{YTTY}\right.$ TGNCCRTAYTTNCKCCA-3', $\mathrm{Y}=\mathrm{C} / \mathrm{T}, \quad \mathrm{R}=\mathrm{A} / \mathrm{G}, \quad \mathrm{K}=$ $\mathrm{G} / \mathrm{T}, \mathrm{N}=\mathrm{A} / \mathrm{G} / \mathrm{C} / \mathrm{T})$ and $5^{\prime}-$ Oligo $(\mathrm{dT}) 20 \mathrm{MN}-3^{\prime}(\mathrm{M}=\mathrm{A} / \mathrm{G} / \mathrm{C}$, $\mathrm{N}=\mathrm{A} / \mathrm{G} / \mathrm{C} / \mathrm{T}$ ) to amplify the conserved regions of the WRKY orthologs based on sequence information from the A. thaliana WRKY gene family in TAIR10 (http:// arabidopsis.org/index.jsp) and the Chinese cabbage chiifu genome in BRAD (http://brassicadb.org/brad/). Based on the results of sequenced polymerase chain reaction (PCR) products from the conserved region of each of the WRKY orthologs and the full length sequences of Chinese cabbage WRKY orthologs (data not shown), we designed gene-specific primers (Additional file 1: Table S1) and performed 5' -and 3'-RACE (Smart RACE cDNA amplification kit; Clontech, Mountain View, CA) to amplify the full-length cDNA sequences of $B c W R K Y \mathrm{~s}$ in the stress-induced Pak-choi cDNA library. The 56 open reading frames (ORFs) from the stress-inducible $B C W R K Y$ cDNA sequences were amplified by reverse transcription-polymerase chain reaction (RT-PCR) using gene-specific primers (Additional file 1: Table S1). PCR reactions included a pre-incubation at $94^{\circ} \mathrm{C}$ for $5 \mathrm{~min}$, followed by 30 cycles of denaturation at $94^{\circ} \mathrm{C}$ for $30 \mathrm{~s}$, annealing at $55-65^{\circ} \mathrm{C}$ for $2 \mathrm{~min}$, and extension at $72^{\circ} \mathrm{C}$ for $10 \mathrm{~min}$. The amplification fragments were cloned in to PMD-19T (Takara). The gene ORF size was confirmed and sequenced using the ABI3730 sequencer (Applied Biosystems, Foster City, CA).
Multiple sequences alignments and phylogenetic analyses The amino acid sequence alignments of putative $B c W R K Y_{\mathrm{S}}$ were performed using ClustalW implemented in the MEGA 5 software [38] with the default settings. They were visualized and manually modified using Jalview 2.7 [39]. Phylogenetic trees of BcWRKY proteins and WRKY domains were built using the maximum likelihood method in MEGA 5. The confidence level of the monophyletic group was estimated using a bootstrap analysis of 1000 replicates.

\section{Identification of conserved motifs}

The 56 putative BcWRKY protein sequences used for the phylogenetic analysis were detected by MEME [40] to analyze possible conserved motifs using the default parameters, except that the maximum number of motifs to identify was defined as eight and the maximum width was set to 200 .

\section{Nuclear localization analysis}

A subcellular localization analysis of deduced BcWRKY proteins was performed by bioinformatics predictions and experimental approaches. NLSs were detected using NLStradamus [41], and nuclear protein scores were calculated separately using WOLF PSORT [42] and NucPred [43] with the default settings. Meanwhile, two expression vectors were constructed (Figure $3 \mathrm{~B}$ ) to investigate the subcellular localizations of BcWRKY TFs using a transient expression system in onion epidermal cells. The full-length coding sequences of BcWRKY25 and BcWRKY4O were amplified using Gateway-specific primers (Additional file 5: Table S2) cloned into an entry vector, and then subcloned into pEarleyGate101 by Gateway technology (Invitrogen, Carlsbad, CA). The yellow fluorescent marker protein (YFP) was fused to BcWRKY25 and BcWRKY40. Gold particles with a diameter of $1 \mu \mathrm{m}$ coated with 35S: BcW25-YFP, 35S:BcW40-YFP, and 35S:YFP (Figure 3B) were introduced into onion epidermal cells using particle bombardment (PDS-100/He particle delivery system; Bio-Rad, Hercules, CA). After incubation at $22^{\circ} \mathrm{C}$ for at least $12 \mathrm{~h}$ under darkness, fluorescence and bright-light images were observed by laser scanning confocal microscopy (Leica, TCS SP2, Wetzlar, Germany).

\section{RNA isolation and quantitative real-time PCR (qPCR)}

Leaf samples were obtained from control and multiple abiotic stress-treated plants for total RNA extraction with an RNA kit (RNAsimply total RNA Kit, Tiangen). Total RNA was treated with DNase I (Takara) for potential genomic DNA contamination. For qPCR analysis, 1 $\mu \mathrm{g}$ of total RNA was used to synthesize the first-strand cDNA using the PrimeScript ${ }^{\text {tm }}$ RT reagent Kit (Takara) for RT-PCR in a $20-\mu$ l reaction volume according to the manufacturer's instructions. The cDNA reaction 
mixture was diluted 1:10 with EASY Dilution for Real Time PCR (Takara), and $2 \mu \mathrm{l}$ was used as the template in the 20- $\mu$ PCR reactions. PCR reactions included a pre-incubation at $95^{\circ} \mathrm{C}$ for $4 \mathrm{~min}$, followed by 40 cycles of denaturation at $95^{\circ} \mathrm{C}$ for $30 \mathrm{~s}$, annealing at $58^{\circ} \mathrm{C}$ for $30 \mathrm{~s}$, and extension at $72^{\circ} \mathrm{C}$ for $30 \mathrm{~s}$. All the reactions were performed in a 7500 Fast Real-Time PCR System (Applied Biosystems) using SYBR ${ }^{\circ}$ Premix Ex Taq (Takara). After the PCR was run, a melting curve $\left(65-95^{\circ} \mathrm{C}\right.$, at increments of $0.5^{\circ} \mathrm{C}$ ) was generated to confirm the specificity of the amplification. The gene glyceraldehydes3-phosphate dehydrogenase $(B c G A P D H)$ was used as an internal control. The gene-specific primers that were used to detect transcripts are listed in Additional file 1: Table S1. The relative gene expression was calculated as previously described [44]. The gene expression was measured from at least three biological replicates (three technical replicates for each biological replicate).

\section{Pearson correlation and co-regulatory networks}

To display the regulatory relationships of stress-inducible $B c W R K Y$ genes that mediated multiple abiotic stresses, the Pearson correlation coefficients of stress-inducible $B c W R K Y$ gene pairs were calculated using a house Perl script based on $\log _{2}$ transformed qPCR data. All of the gene pairs whose Pearson coefficient was significant at the 0.05 significance level ( $\mathrm{p}$-value) were collected for a gene co-regulatory network analysis. Co-expression networks were graphically visualized using Cytoscape version 2.8 [45] based on Pearson correlation coefficients of $B c W R K Y$ gene pairs. The nodes represent genes and the edges between nodes represent gene pairs expressing correlations (hypothetical interactions). The different edge line styles indicate different correlation levels between corresponding nodes, which, in turn, indicate different interaction strengths between the co-regulated gene pairs. In addition, we compared the protein-protein relationships of stress-inducible BcWRKY orthologs in Arabidopsis using STRING 9 (http://string.embl.de/) with the default program parameter settings.

\section{Additional files}

Additional file 1: Table S1. Primers used for RT-PCR and real-time PCR. Additional file 2: Figure S1. Alignment of WRKY domains between Pak-choi and Arabidopsis.

Additional file 3: Table S3. Pearson correlation coefficient of stress-induced BCWRKYS.

Additional file 4: Figure S2. A confidence co-regulatory network of WRKY orthologs in Arabidopsis.

Additional file 5: Table S2. Gatway cloning primers for subcelluar localization.

\section{Competing interests}

The authors declare that they have no competing interests.

\section{Authors' contributions}

$J T, F W$, and XH conceived the project. JT, ZW, and ZH prepared the plant materials and carried out the gene cloning and expression analyses. JT and FW performed analyzed the data; AX provided advice on protein classification. JT and XH prepared the manuscript. FW, AX and XH revised and proofread the manuscript. All authors read and approved the final manuscript.

\section{Acknowledgements}

This study was supported by the National Program on Key Basic Research Projects of China (2012CB113900), the National High Tech Research Development Program of China (2012AA100100), and the National Natural Science Foundation of China (31201633) as well as the Priority Academic Program Development of Jiangsu Higher Education Institutions.

Received: 6 August 2013 Accepted: 22 November 2013

Published: 25 November 2013

\section{References}

1. Xiong L, Ishitani M, Zhu J: Interaction of osmotic stress, temperature, and abscisic acid in the regulation of gene expression in Arabidopsis. Plant Physiol 1999, 119(1):205-212.

2. Chinnusamy V, Zhu J, Zhu J-K: Cold stress regulation of gene expression in plants. Trends Plant Sci 2007, 12(10):444-451.

3. Chen WJ, Zhu T: Networks of transcription factors with roles in environmental stress response. Trends Plant Sci 2004, 9(12):591-596.

4. Eulgem T, Rushton PJ, Robatzek S, Somssich IE: The WRKY superfamily of plant transcription factors. Trends Plant Sci 2000, 5(5):199-206.

5. Rushton PJ, Somssich IE, Ringler $P$, Shen QJ: WRKY transcription factors. Trends Plant Sci 2010, 15(5):247-258

6. Chen L, Song Y, Li S, Zhang L, Zou C, Yu D: The role of WRKY transcription factors in plant abiotic stresses. Biochim Biophys Acta 2012, 1819(2):120-128.

7. Yamasaki K, Kigawa T, Inoue M, Tateno M, Yamasaki T, Yabuki T, Aoki M, Seki E, Matsuda T, Tomo Y, et al: Solution structure of an Arabidopsis WRKY DNA binding domain. Plant Cell Online 2005, 17(3):944-956.

8. Xie Z, Zhang Z-L, Zou X, Huang J, Ruas P, Thompson D, Shen QJ: Annotations and functional analyses of the rice WRKY gene superfamily reveal positive and negative regulators of Abscisic acid signaling in Aleurone cells. Plant Physiol 2005, 137(1):176-189.

9. Mangelsen E, Kilian J, Berendzen KW, Kolukisaoglu UH, Harter K, Jansson C, Wanke D: Phylogenetic and comparative gene expression analysis of barley (Hordeum vulgare) WRKY transcription factor family reveals putatively retained functions between monocots and dicots. BMC Genomics 2008, 9:194.

10. Ling J, Jiang W, Zhang Y, Yu H, Mao Z, Gu X, Huang S, Xie B: Genome-wide analysis of WRKY gene family in Cucumis sativus. BMC Genomics 2011, 12(1):471.

11. Huang S, Gao Y, Liu J, Peng X, Niu X, Fei Z, Cao S, Liu Y: Genome-wide analysis of WRKY transcription factors in Solanum lycopersicum. Mol Genet Genomics 2012, 287(6):495-513.

12. $L i H L$, Zhang LB, Guo D, Li CZ, Peng SQ: Identification and expression profiles of the WRKY transcription factor family in Ricinus communis. Gene 2012, 503(2):248-253.

13. Tripathi $P$, Rabara RC, Langum TJ, Boken AK, Rushton DL, Boomsma DD, Rinerson Cl, Rabara J, Reese RN, Chen X, et al: The WRKY transcription factor family in Brachypodium distachyon. BMC Genomics 2012, 13(1):270.

14. Wei KF, Chen J, Chen YF, Wu LJ, Xie DX: Molecular phylogenetic and expression analysis of the complete WRKY transcription factor family in maize. DNA Res 2012, 19(2):153-164.

15. Xiong W, Xu X, Zhang L, Wu P, Chen Y, Li M, Jiang H, Wu G: Genome-wide analysis of the WRKY gene family in physic nut (Jatropha curcas L). Gene 2013, 524(2):124-132.

16. Scarpeci TE, Zanor MI, Valle EM: The WRKY30 transcription factor: a node of convergence for abiotic and biotic signals in plant defence. Plant Biology (Rockville) 2009, 2009(Suppl. S):146.

17. Shang Y, Yan L, Liu Z-Q, Cao Z, Mei C, Xin Q, Wu F-Q, Wang X-F, Du S-Y, Jiang $\mathrm{T}$, et al: The Mg-Chelatase $\mathrm{H}$ subunit of Arabidopsis antagonizes a group of WRKY transcription repressors to relieve ABA-responsive genes of inhibition. Plant Cell 2010, 22:1909-1935.

18. Antoni R, Rodriguez L, Gonzalez-Guzman M, Pizzio GA, Rodriguez PL: News on $A B A$ transport, protein degradation, and $A B F s / W R K Y s$ in $A B A$ signaling. Curr Opin Plant Biol 2011, 14(5):547-553. 
19. Liu ZQ, Yan L, Wu Z, Mei C, Lu K, Yu YT, Liang S, Zhang XF, Wang XF, Zhang DP: Cooperation of three WRKY-domain transcription factors WRKY18, WRKY40, and WRKY60 in repressing two ABA-responsive genes ABI4 and ABI5 in Arabidopsis. J Exp Bot 2012, 63(18):6371-6392.

20. Van Aken $O$, Zhang B, Law S, Narsai R, Whelan J: AtWRKY40 and AtWRKY63 modulate the expression of stress-responsive nuclear genes encoding mitochondrial and chloroplast proteins. Plant Physiol 2013, 162(1):254-271.

21. Jiang $Y$, Deyholos MK: Functional characterization of Arabidopsis $\mathrm{NaCl}$ inducible WRKY25 and WRKY33 transcription factors in abiotic stresses. Plant Mol Biol 2009, 69(1-2):91-105.

22. Ren X, Chen Z, Liu Y, Zhang H, Zhang M, Liu Q, Hong X, Zhu J-K, Gong Z: $\mathrm{ABO} 3$, a WRKY transcription factor, mediates plant responses to abscisic acid and drought tolerance in Arabidopsis. Plant J 2010, 63(3):417-429.

23. Zou CS, Jiang WB, Yu DQ: Male gametophyte-specific WRKY34 transcription factor mediates cold sensitivity of mature pollen in Arabidopsis. J Exp Bot 2010, 61(14):3901-3914.

24. Tao Z, Kou Y, Liu H, Li X, Xiao J, Wang S: OsWRKY45 alleles play different roles in abscisic acid signalling and salt stress tolerance but similar roles in drought and cold tolerance in rice. J Exp Bot 2011, 62(14):4863-4874.

25. Hu Y, Chen L, Wang H, Zhang L, Wang F, Yu D: Arabidopsis transcription factor WRKY8 functions antagonistically with its interacting partner VQ9 to modulate salinity stress tolerance. Plant J 2013, 74(5):730-745.

26. Wang F, Hou X, Tang J, Wang Z, Wang S, Jiang F, Li Y: A novel coldinducible gene from Pak-choi (Brassica campestris ssp. chinensis), BcWRKY46, enhances the cold, salt and dehydration stress tolerance in transgenic tobacco. Mol Biol Rep 2012, 39(4):4553-4564.

27. Usadel B, Obayashi T, Mutwil M, Giorgi FM, Bassel GW, Tanimoto M, Chow A, Steinhauser D, Persson S, Provart NJ: Co-expression tools for plant biology: opportunities for hypothesis generation and caveats. Plant Cell Environ 2009, 32(12):1633-1651.

28. Movahedi S, Van Bel M, Heyndrickx KS, Vandepoele K: Comparative co-expression analysis in plant biology. Plant Cell Environ 2012, 35(10):1787-1798.

29. Bordych C, Eisenhut M, Pick TR, Kuelahoglu C, Weber APM: Co-expression analysis as tool for the discovery of transport proteins in photorespiration. Plant Biol 2013, 15(4):686-693.

30. Berri S, Abbruscato P, Faivre-Rampant O, Brasileiro AC, Fumasoni I, Satoh K, Kikuchi S, Mizzi L, Morandini P, Pe ME, et al: Characterization of WRKY co-regulatory networks in rice and Arabidopsis. BMC Plant Biol 2009, 9:120

31. Jiang FL, Wang F, Wu Z, Li Y, Shi GJ, Hu JD, Hou XL: Components of the Arabidopsis CBF cold-response pathway are conserved in non-heading Chinese cabbage. Plant Mol Biol Report 2011, 29(3):525-532.

32. Wang $X$, Wang $H$, Wang J, Sun R, Wu J, Liu S, Bai Y, Mun JH, Bancroft I, Cheng $F$, et al: The genome of the mesopolyploid crop species Brassica rapa. Nat Genet 2011, 43(10):1035-1039.

33. Ülker B, Somssich IE: WRKY transcription factors: from DNA binding towards biological function. Curr Opin Plant Biol 2004, 7(5):491-498.

34. Ciolkowski I, Wanke D, Birkenbihl RP, Somssich IE: Studies on DNA-binding selectivity of WRKY transcription factors lend structural clues into WRKY-domain function. Plant Mol Biol 2008, 68(1-2):81-92.

35. Yamasaki K, Kigawa T, Seki M, Shinozaki K, Yokoyama S: DNA-binding domains of plant-specific transcription factors: structure, function, and evolution. Trends Plant Sci 2013, 18(5):267-276.

36. Mochida K, Uehara-Yamaguchi Y, Yoshida T, Sakurai T, Shinozaki K: Global landscape of a co-expressed gene network in barley and its application to gene discovery in Triticeae crops. Plant Cell Physiol 2011, 52(5):785-803.

37. Chi Y, Yang Y, Zhou Y, Zhou J, Fan B, Yu JQ, Chen Z: Protein-protein interactions in the regulation of WRKY transcription factors. Mol Plant 2013, 6(2):287-300

38. Tamura K, Peterson D, Peterson N, Stecher G, Nei M, Kumar S: MEGA5: molecular evolutionary genetics analysis using maximum likelihood, evolutionary distance, and maximum parsimony methods. Mol Biol Evol 2011, 28(10):2731-2739.

39. Waterhouse AM, Procter JB, Martin DMA, Clamp M, Barton GJ: Jalview version 2-a multiple sequence alignment editor and analysis workbench. Bioinformatics 2009, 25(9):1189-1191.

40. Bailey TL, Boden M, Buske FA, Frith M, Grant CE, Clementi L, Ren J, Li WW, Noble WS: MEME SUITE: tools for motif discovery and searching. Nucleic Acids Res 2009, 37(Web Server issue):W202-W208.
41. Nguyen Ba A, Pogoutse A, Provart N, Moses A: NLStradamus: a simple Hidden Markov model for nuclear localization signal prediction. BMC Bioinforma 2009, 10(1):202.

42. Horton P, Park K-J, Obayashi T, Fujita N, Harada H, Adams-Collier CJ, Nakai K. WoLF PSORT: protein localization predictor. Nucleic Acids Res 2007, 35(suppl 2):W585-W587.

43. Brameier M, Krings A, MacCallum RM: NucPred-predicting nuclear localization of proteins. Bioinformatics 2007, 23(9):1159-1160.

44. Livak KJ, Schmittgen TD: Analysis of relative gene expression data using real-time quantitative $P C R$ and the $2-\Delta \Delta C T$ method. Methods 2001 25(4):402-408.

45. Smoot ME, Ono K, Ruscheinski J, Wang P-L, Ideker T: Cytoscape 2.8 new features for data integration and network visualization. Bioinformatics 2011, 27(3):431-432.

doi:10.1186/1471-2229-13-188

Cite this article as: Tang et al: Characterization and co-expression analysis of WRKY orthologs involved in responses to multiple abiotic stresses in Pak-choi (Brassica campestris ssp. chinensis). BMC Plant Biology 2013 13:188.

\section{Submit your next manuscript to BioMed Central and take full advantage of:}

- Convenient online submission

- Thorough peer review

- No space constraints or color figure charges

- Immediate publication on acceptance

- Inclusion in PubMed, CAS, Scopus and Google Scholar

- Research which is freely available for redistribution 